\title{
A patient-level key performance indicator set to measure the effectiveness of fracture liaison services and guide quality improvement: a position paper of the IOF Capture the Fracture Working Group, National Osteoporosis Foundation and Fragility Fracture Network
}

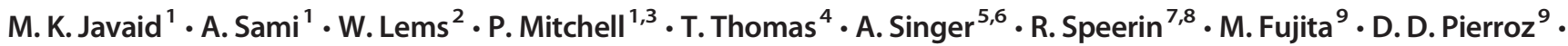 \\ K. Akesson $^{10}$. P. Halbout ${ }^{9}$ - S. Ferrari ${ }^{11}$ - C. Cooper ${ }^{1,12}$
}

Received: 14 January 2020 / Accepted: 3 March 2020/Published online: 8 April 2020

(C) The Author(s) 2020

\begin{abstract}
Summary The International Osteoporosis Foundation (IOF) Capture the Fracture ${ }^{\circledR}$ Campaign with the Fragility Fracture Network (FFN) and National Osteoporosis Foundation (NOF) has developed eleven patient-level key performance indicators (KPIs) for fracture liaison services (FLSs) to guide quality improvement.

Introduction Fracture Liaison Services (FLSs) are recommended worldwide to reduce fracture risk after a sentinel fracture. Given not every FLS is automatically effective, the IOF Capture the Fracture working group has developed and implemented the Best Practice Framework to assess the organisational components of an FLS. We have now developed a complimentary KPI set that extends this assessment of performance to the patient level.

Methods The Capture the Fracture working group in collaboration with the Fragility Fracture Network Secondary Fragility Fracture Special Interest Group and National Osteoporosis Foundation adapted existing metrics from the UK-based Fracture Liaison Service Database Audit to develop a patient-level KPI set for FLSs.

Results Eleven KPIs were selected. The proportion of patients: with non-spinal fractures; with spine fractures (detected clinically and radiologically); assessed for fracture risk within 12 weeks of sentinel fracture; having DXA assessment within 12 weeks of sentinel fracture; having falls risk assessment; recommended anti-osteoporosis medication; commenced of strength and balance exercise intervention within 16 weeks of sentinel fracture; monitored within 16 weeks of sentinel fracture; started antiosteoporosis medication within 16 weeks of sentinel fracture; prescribed anti-osteoporosis medication 52 weeks after sentinel fracture. The final KPI measures data completeness for each of the other KPIs. For these indicators, levels of achievement were set at the $<50 \%, 50-80 \%$ and $>80 \%$ levels except for treatment recommendation where a level of $50 \%$ was used.
\end{abstract}

M. K. Javaid

Kassim.javaid@ndorms.ox.ac.uk

1 The Botnar Research Centre, Nuffield Department of Orthopaedics, Rheumatology and Orthopaedic Sciences, University of Oxford, Oxford OX4 7LD, UK

2 VU University Medical Center, Amsterdam, The Netherlands

3 School of Medicine, Sydney Campus, The University of Notre Dame Australia, 140 Broadway, Sydney, NSW 2007, Australia

4 Department of Rheumatology, Hôpital Nord, CHU de Saint-Etienne, and INSERM U1059, University of Lyon, Saint-Etienne, France

5 Department of Medicine, MedStar Georgetown University Hospital and Georgetown University Medical Center, Washington, DC, USA
6 Department of Obstetrics and Gynecology, MedStar Georgetown University Hospital and Georgetown University Medical Center, Washington, DC, USA

7 Fragility Fracture Network, Zürich, Switzerland

8 Musculoskeletal Network, NSW Agency for Clinical Innovation, Chatswood, Australia

9 International Osteoporosis Foundation, Nyon, Switzerland

10 Department of Orthopaedics, Skane University Hospital, Malmö, Sweden

11 Division of Bone Disease, Department of Internal Medicine Specialties, Faculty of Medicine, Geneva University Hospital, Geneva, Switzerland

12 MRC Lifecourse Epidemiology Unit, University of Southampton, Southampton, UK 
Conclusion This KPI set compliments the existing Best Practice Framework to support FLSs to examine their own performance using patient-level data. By using this KPI set for local quality improvement cycles, FLSs will be able to efficiently realise the full potential of secondary fracture prevention and improved clinical outcomes for their local populations.

Keywords Osteoporosis · Secondary fracture prevention · Fracture Liaison Service/FLS · Quality improvement · Key performance indicators

\section{Introduction}

The aging demographic poses a major threat to healthcare systems with absolute increases in the burden of noncommunicable diseases, including osteoporosis, with 9 million fragility fractures every year $[1,2]$. Fragility fractures are robust predictors of sustaining future fractures [3], and this has led to the global prioritisation of systematic secondary fracture prevention based on expected clinical and cost effectiveness [4-8].

Despite a wide range of effective anti-osteoporosis medications and a growing elderly population, the proportion of patients receiving adequate secondary fracture prevention is falling $[9,10]$. In some countries, this is associated with a plateau in the previously improved trends in fracture rates [11]. International societies [4-8] have recommended specialised systems of healthcare delivery called fracture liaison services (FLSs) to close this care gap [12]. An FLS is a small team of healthcare professionals that identifies, investigates, recommends treatment and monitors over time patients aged 50 and above presenting with a fragility fracture [13]
(Fig. 1). Systematic reviews of FLS have demonstrated their potential clinical [14] and cost effectiveness [15].

FLSs often operate in complex local healthcare systems relying on the effective interplay between people, equipment, processes and institutions as well as competing priorities, resources and reimbursement. Most complex services require active service improvement to adapt the service to become effective and sustainable based on local healthcare characteristics; one size does not fit all [16]. A key component of service improvement is the availability of indicators that reflect the performance of the service and can subsequently be used to highlight areas requiring improvement as well as measure the impact of changes in service delivery. The Capture the Fracture Best Practice Framework (BPF) assesses FLSs at the organisational level [17]. It includes 13 standards measuring scope of FLS within bronze, silver and gold levels. The BPF has been implemented globally [18] with over 380 FLSs now participating (https://www.capturethefracture.org/map-ofbest-practice). The BPF was designed to measure the scope of FLSs in terms of organisational characteristics,
Fig. 1 Fracture liaison service overall pathway

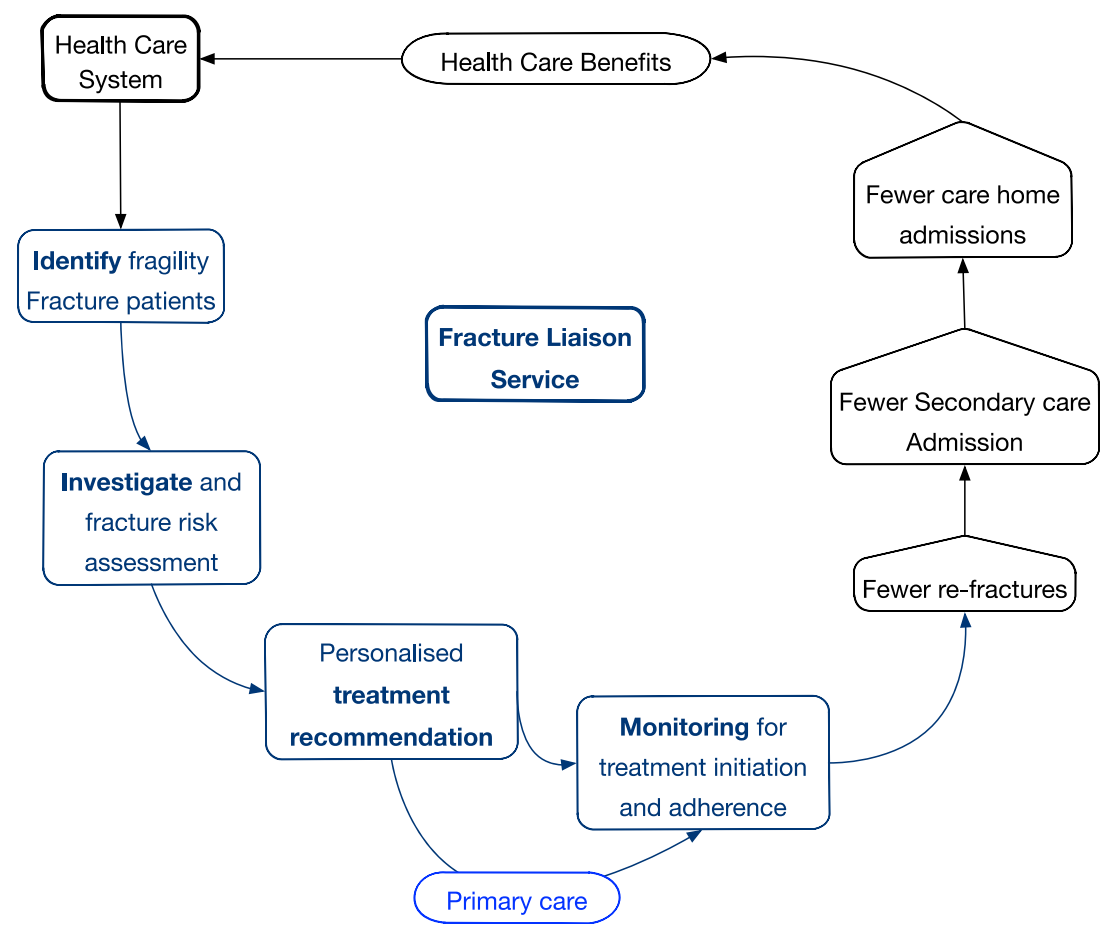




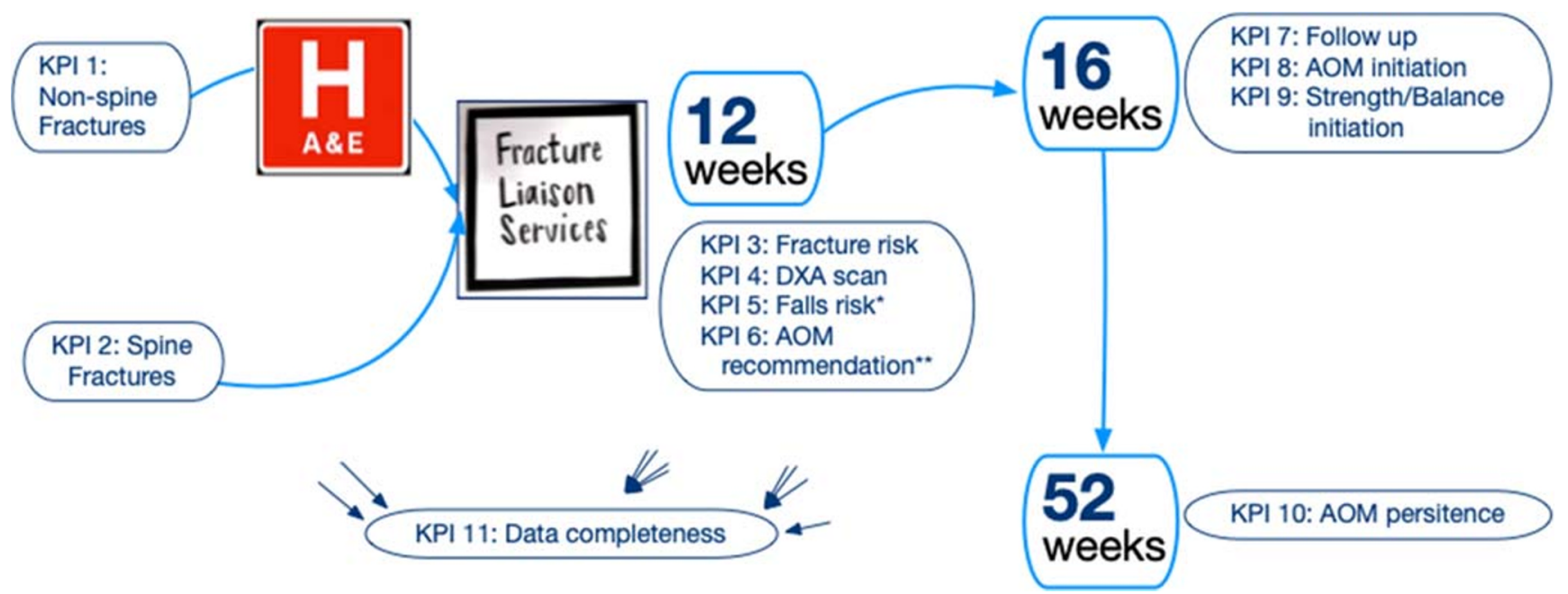

Fig. 2 Mapping the 11 key performance indicators to the broad patient pathway following a fragility fracture

and overall rates of identification, assessment, falls assessment and types of treatment, communication and follow-up. These standards are helpful for identifying major gaps in the service delivery such as types of patients with fractures identified and presence of monitoring. They are less helpful for helping established FLSs reach their full potential for secondary fracture prevention in the context of local challenges and opportunities. We here present a complimentary key performance indicator (KPI) set that measures the realworld secondary fracture prevention delivery at the patient level. The objective of these KPIs is to demonstrate areas for service improvement and measure the impact of service interventions within a plan-do-study-act methodology, the building blocks of iterative healthcare improvement [16].

\section{Methods}

The Capture the Fracture (CtF) working group, International Osteoporosis Foundation, worked with the Fragility Fracture Network Secondary Fragility Fracture Special Interest Group (RS) and National Osteoporosis Foundation (AS), to adapt an existing KPI set from the UK-based Fracture Liaison Service Database (FLSDB) Audit [19, 20] for international use. The aim of the KPI set was to identify specific indicators an FLS should record that would be used for service improvement. The KPI set went through an initial round for comment and then a final round for consensus.

To allow an FLS to compare their service delivery across indicators, levels of achievement were set as green (adequate), amber (needs improving) and red (priority area for service improvement). Achievement of these indicators could be used to compare the performance of an FLS over time or between FLSs at regional or national levels. Once consensus had been reached, a table was created to describe the specific information an FLS should record to be able to complete the KPIs.

\section{Results}

Eleven indicators were developed from the FLSDB set and are described below in terms of numerator, denominator and key points for clarification (Fig. 2), with a table of specific information needed to complete them (Table 1).

\section{Indicator 1: Identification of patients with non-spine fragility fractures}

Numerator: Total number of patients with non-spine fragility fractures identified

Denominator: Expected local non-spine fragility fracture caseload

Identifying all patients with a non-spine fragility fracture is the first step for an FLS. How an FLS identifies adults with a non-spine fracture will vary depending on local, regional and national healthcare systems and reporting. Specifically, a patient shall be considered identified for the purpose of this position paper, once the identity of the patient becomes known to the FLS service, whether this results from the FLS service being physically embedded in a fracture clinic or ward or whether patient details are forwarded in the form of patient lists or shared electronically in an EPR system. A non-spine fragility fracture excludes fractures of the face, skull, scaphoid and digits. There are numerous methods to estimate the expected caseload of non-spine fragility fractures for the denominator. The preferred method is reliable local data of all patients with non-spine fragility fractures attending the local health services. If this is not available, an estimate of the fragility fracture caseload can be made from population- 


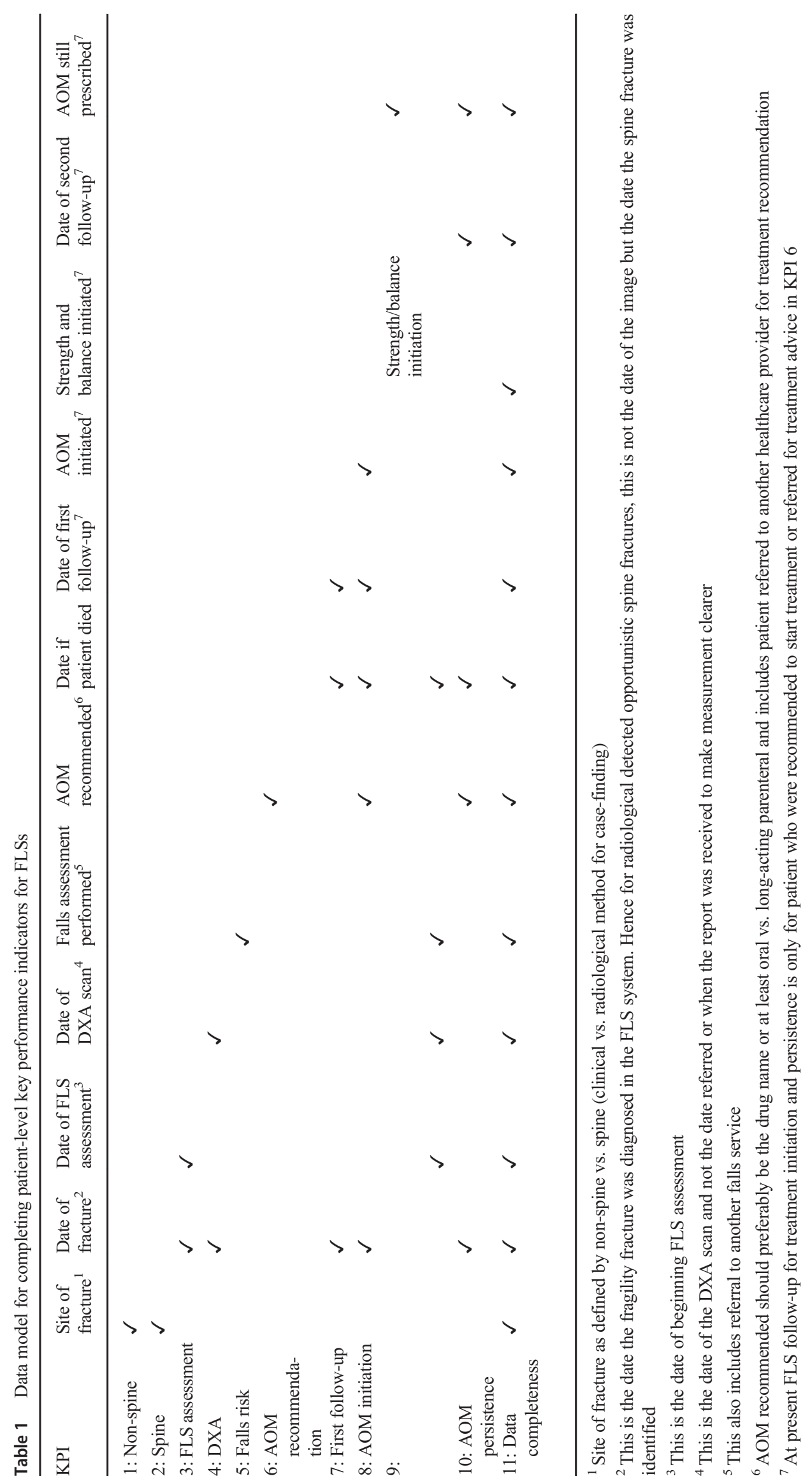


based incidence data or by multiplying by 5 the number of hip fractures [21], recognising that there will be a yearly variation in the number of hip fractures seen at a site. The levels of achievement were set at red (0-49\% of expected caseload), amber (50-79\%) and green (80\% or greater) for this indicator. This reflects the number of patients identified by the FLS and not the number invited to attend the FLS. It is expected that for a significant minority, especially in the elderly, attendance to an FLS would be clinically inappropriate. The proportion for whom FLS is clinically inappropriate is measured by KPI 6 .

\section{Indicator 2: Identification patients with spine fractures}

Numerator: Number of patients with a spine fracture identified Denominator: Number of hip fracture patients

A separate indicator for spine fractures was included because spine fractures are both common [22] and a powerful predictor of imminent fracture risk [23], and the care pathway for identifying spine fractures is critically different from other fractures. Most patients with spine fractures are managed outside the trauma-orthopaedic setting [24]. Patients may present with symptoms such as back pain or height loss, or be subclinical and detected from opportunistic screening using VFA $[25,26]$ or radiological reports [27] or images. Debate on the specific radiological characteristics for spine fractures has led to a degree of uncertainty of the expected caseload of spine fractures for an FLS [28]. How an FLS identifies eligible patients with spine fractures will vary by national, regional and local healthcare systems and reporting. Specifically, a patient with a spine fracture shall be considered identified, for the purpose of this position paper, once the identity of the patient becomes known to the FLS service, whatever method of identification is used. The number of clinical spine fractures detected by an FLS is expected to be $75 \%$ of the local number of patients with a hip fracture [29]. The number of radiological opportunistically detected spine fractures detected by an FLS is provisionally set using expert consensus at twice the number of clinical spine fractures and will be reviewed regularly depending on the published evidence. The levels of achievement for red, amber and green reflect achieving $0-49 \%, 50-79 \%$ and $80 \%$ or higher of this estimate respectively.

\section{Indicator 3: Initial investigation including fracture risk assessment within 12 weeks}

Numerator: Number of patients assessed within 12 weeks of the sentinel fracture

Denominator: Total number of patients identified

Appreciation of the imminent fracture risk following an incident fracture in the FLS setting requires rapid assessment to identify patients meeting treatment thresholds
[30]. Ideally fracture risk assessment should be part of the initial management plan to treat the sentinel fracture; this also may reduce non-attendance rates [31] and improve treatment rates [32].

Investigation or assessment is a multistage process including patient history, physical examination, laboratory testing and imaging. The time of assessment is defined as date this process starts in those identified by KPI 1 or 2 above, for example first contact by the FLS. The levels of achievement for red, amber and green reflect achieving 0-49\%, 50-79\% and $80 \%$ or higher of this indicator respectively.

While fracture risk assessment often includes DXA, with or without VFA [33], we recognise that DXA may not be required in all patients (e.g. the very frail, those with a hip fragility fracture), and so DXA has been included in a separate indicator.

\section{Indicator 4: DXA within 12 weeks}

Numerator: Number of patients with date of DXA within 12 weeks of the sentinel fracture

Denominator: Number of patients for whom DXA is recommended according to regional or national guidelines

DXA can be used to risk stratify patients into high vs. low risk of subsequent fracture [34] as well as a baseline measure for future comparative measurements [35]. In the post-fracture setting, however, DXA may not be mandatory for patients aged 75 years and older [36] or those with fragility fractures of the hip and spine [37]. In the latter setting, treatment should not be delayed while waiting to acquire a DXA measurement. Further, the requirement for DXA will differ by regional/ national practice and anti-osteoporosis medication reimbursement criteria for or time since last DXA, e.g. within the last 24 months. These factors should be reflected in the denominator. The levels of achievement for red, amber and green reflect achieving $0-49 \%, 50-79 \%$ and $80 \%$ or higher of this indicator respectively.

\section{Indicator 5: Falls risk assessment}

Numerator: Number of patients with a falls assessment or screen performed, recommended, or referred to or already under a falls service

Denominator: Total number of patients identified

The aim of an FLS is to reduce re-fracture risk and effective falls prevention interventions complement use of antiosteoporosis medications in individuals with a fracture. This includes both non-spine fractures and spine fractures as both increase falls risk [38]. The type of falls assessment range from tools that rapidly assess falls risk in the office [39] to a full multifactorial fall risk assessment including tests of balance and strength, such as timed up and go test [40]. The content of the falls assessment for patients identified by the 
FLS should be based on local, regional and national recommendations and completed in patients presenting with a fragility fracture. Most falls services are typically aimed at patients aged 65 years and older but some degree of falls assessment is still required in those aged 50 to 64 years, with those identified at high falls risk referred onward to appropriate services. Additionally, in some healthcare settings, falls assessment is provided by services/departments that are different to the FLS. In these situations, FLSs are recommended to use a 'referred to falls' option. No specific deadline is given and it is recommended that falls assessments are commenced as soon as patient is deemed fit enough to participate. Timing of falls assessment will be captured, in part, through indicator 9 related to initiation of strength and balance classes. The levels of achievement for red, amber and green reflect achieving $0-49 \%, 50-79 \%$ and $80 \%$ or higher of this indicator respectively.

\section{Indicator 6: Anti-osteoporosis medication (AOM) recommended as appropriate}

Numerator: Number of patients with a treatment recommendation as clinical decision to treat

Denominator: Total number of patients identified

Ensuring enough high-risk patients are recommended $\mathrm{AOM}$ is integral to reducing secondary fractures at the population level. The reason why we have focused on proportion recommended AOM rather than simply a treatment decision being made is that previous work has demonstrated that even with the same national treatment guideline, there was unexplained variation of rates of treatment recommendation between FLSs [20]. Recognising that many FLS are delivered by nurses or allied health professionals with limited prescribing rights or ability to recommend medications, the numerator also includes patients where the FLS has referred patients to a clinician for AOM recommendation. No timeline has been required as initiation of anti-osteoporosis medication in KPI 8 will be used as a measure of timely recommendations. The denominator includes patients who were already on an AOM at the time of fracture given the commonest reason for a fracture on treatment is non-adherence. The numerator includes all patients who are recommended treatment after a fragility fracture irrespective of treatment status at the time of fracture and so includes patients who are advised to continue with the same anti-osteoporosis treatment before the index fracture in line with IOF guidelines [35].

The expected level of achievement for this indicator will depend on the local risk profile of the population in terms of comorbidity, prevalence of contra-indications and interpretation of the risks vs. benefits of therapy in certain populations (e.g. those with very limited lifespan [41]) and national/ regional guidelines on fracture risk assessment tools (such as FRAX [3] and Garvin [42]) and thresholds for interventions [6]. If the proportion of patients recommended treatment is low, this may reflect inappropriately high treatment thresholds, local differences in the type of fractures seen, e.g. FLS serving institutions with specialised ankle or foot services, or the FLS is missing a cohort of high-risk patients. The level of achievement for this indicator has been set at $50 \%$ or more patients recommended or referred for AOM, based on national audit data [20].

\section{Indicator 7: Recorded follow-up within 16 weeks post index fracture}

Numerator: Number of patients followed up post sentinel fracture

Denominator: Total number of patients referred or recommended anti-osteoporosis medication (AOM) minus patients who have died

Given the imminent risk of fracture, it is important that patients have an early follow-up to ensure anti-osteoporosis treatment recommendations have been implemented soon after the sentinel fracture. The denominator only includes patients referred or recommended AOM and not those only recommend calcium/vitamin D recommendations or nonpharmacological approaches. A variety of methods can be used to monitor patients including face to face clinic assessments, telehealth (remote) visits, postal questionnaires, telephone consultations and email, and should be tailored to local practice and patient needs. Patients given a long-acting parenteral AOM after fracture may still require follow-up to check for unwanted effects, adherence to calcium and vitamin D supplements and scheduling for subsequent treatments. The levels of achievement for red, amber and green reflect achieving $0-49 \%, 50-79 \%$ and $80 \%$ or higher of this indicator respectively.

\section{Indicator 8: Commenced anti-osteoporosis medication (AOM) by 16 weeks post index fracture}

Numerator: Number of patients commenced or continuing AOM within 16 weeks of date of fracture

Denominator: Number of patients with a treatment recommendation to start AOM or referred to GP or referred to another clinician minus patients who have died

Treatment initiation can be assessed by patient self-report, clinical review or prescription data. Patients given a longacting parenteral AOM after fracture would automatically have initiated therapy. The levels of achievement for red, amber and green reflect achieving $0-49 \%, 50-79 \%$ and $80 \%$ or higher of this indicator respectively. 


\section{Indicator 9: Strength and balance commenced within 16 weeks of fracture}

Numerator: Number of patients initiating an evidenced based strength and balance class within 16 weeks of the date of fracture according to regional or national guidelines

Denominator: Number of patients with a falls assessment performed, recommended, referred for less those already under falls service minus patients who have died

The numerator specifically only includes evidencebased strength and balance exercise classes that have been shown to reduce falls risk, characterised by group and home classes with progressive resistance training [43]. Care must be taken to ensure the evidence-based exercise is provided by qualified staff and patients are supported to ensure optimal adherence. The denominator was selected as patients who could be eligible for strength and balance classes and should be interpreted with KPI 5. The levels of achievement for red, amber and green reflect achieving $0-49 \%, 50-79 \%$ and $80 \%$ or higher of this indicator respectively.

\section{Indicator 10: Patients taking anti-osteoporosis medication $\mathbf{5 2}$ weeks after the sentinel fracture}

Numerator: Number of patients still taking AOM 52 weeks after the date of sentinel fracture

Denominator: Number of patients with a treatment recommendation to start AOM or referred to GP or referred to another clinician minus patients who have died

Given the known poor adherence to both oral and injectable therapies in the real-world setting, ensuring longer term adherence is vital for an FLS to be effective. Comprehensive assessment of adherence requires assessment of patient taking the anti-osteoporosis medication in accordance with an agreed treatment plan with their clinician [44]. Assessment from patient self-report or prescribing data is reasonable pending evidence for more reliable biomarkers of anti-osteoporosis effect in the early and medium post-fracture period for oral therapies are developed [45]. The levels of achievement for red, amber and green reflect achieving $0-49 \%, 50-79 \%$ and $80 \%$ or higher of this indicator respectively.

\section{Indicator 11: Data completeness}

Numerator: Number of KPIs 1-10 with more than $80 \%$ complete data

Denominator: $10 \mathrm{KPIs}$

Incomplete data collection limits the interpretation of the KPI as the status of patients with missing data is unknown. This indicator measures how many of the above indicators have at least $80 \%$ complete data. It may be possible to extract this data from routine healthcare data and existing registries in some countries. The data for this indicator could form part of the standard FLS assessment and so facilitate the extraction of these KPI from a clinical database. The standards are set at red (0-4 indicators), amber (5-7 indicators) and green (8-10 indicators).

\section{Discussion}

These proposed 11 FLS KPIs provide a composite snapshot of the FLS's delivery of secondary fracture prevention at the patient level. The levels of achievement reflect those used by economic models to demonstrate the expected benefits from secondary fracture prevention in the local setting. Achieving a green level of achievement across all KPIs is needed for an FLS to realise its expected potential to reduce re-fracture rates through secondary fracture prevention. FLSs should actively engage in quality improvement until this is achieved. We recognise for some localities, achieving these KPIs may depend on local healthcare systems that are beyond the remit of the FLS to adapt. These KPIs should be seen as tools for improving service delivery within existing resources as well as describe to payers the precise service gaps present and proposed targets in outcomes for improvement.

The ultimate goal of an FLS is to reduce secondary fracture rates [12]. This essential outcome is needed to demonstrate value to patients, their families, health and social care systems as well as justify sustainable support and engagement by payers and policy makers. The expected number of fractures an FLS will prevent is based on effective identification, investigation, initiation and monitoring. Realizing the health and economic benefits from FLSs depends on consistent achievement of these steps. Robust real-world evidence of the effectiveness of FLSs using fracture events is challenging and takes longer to demonstrate [46]. Using these indicators as surrogate markers will help make sure existing programmes to achieve the goals of their programme. These indicators underlie the importance of each component of secondary fracture prevention for an FLS.

The IOF Capture the Fracture Best Practice Framework [17] lays out the organisational scope of an FLS and expected components for the care pathway. The framework has been successfully used in both national [47] and global [18] settings. The next step for an FLS is to engage in local service improvement to ensure effective and efficient use of FLS resources. Important features of service improvement methodology in healthcare are performance outcomes and balancing measures to inform and drive service improvement [16]. The proposed KPI provides template for FLSs across the globe to use. FLSs also need the capability and capacity to deliver effective service improvement and, if needed, additional training and resources; service improvement has limits. Where 
delivery to these KPIs exceeds the commissioned capacity for an FLS, then additional resources will need to be allocated if the FLS is to deliver the expected reduction in re-fractures.

The individual components for secondary fracture prevention, case-finding, risk assessment, bone and falls prevention interventions and monitoring, are well described [48-50]. Methods to stratify eligibility for AOM are validated nationally and internationally [3,51-54]. AOM use reduces fracture risk by $20-86 \%[55,56]$. Falls prevention strategies, when given to specific at-risk patient groups, significantly reduce falls [43]. The indicators for monitoring treatment adherence, recurrent fractures and falls have been identified $[17,57]$.

The first step for an FLS is to identify patients (both men and women) with a recent fragility fracture. The inclusion criteria may vary by region around age, level of trauma and site of fracture. The recommended age range for an FLS is 50 years and over with no upper limit, as even the elderly with established osteoporosis are more likely to survive long enough to sustain a subsequent fracture and benefit from AOM [41]. An FLS study comparing re-fracture rates in those aged over 85 years has questioned whether the competing risk of death substantially diminishes the capacity of an FLS to reduce fracture rates [58]. However, for elderly women aged 80 years or over, the risk of hip fracture in 5 years is high despite the competing risk of mortality $[41,59]$. The level of trauma includes fractures resulting from a force equivalent to a fall from standing height or less [60]. Fractures of the digits, face, skull and scaphoid are typically excluded as are periprosthetic fractures and those secondary to local cancer involvement or other metabolic bone diseases such as osteogenesis imperfecta or Paget's disease of bone.

Most patients with reported spine fractures do not trigger secondary fracture prevention $[61,62]$. Methods are being developed to improve identification of patients with spine fractures. This may involve text analysis of medical records or radiology reports [27]. While these methods are specific, they may lack sensitivity as they rely on the radiologist reporting the spine fracture, which is known to be poor [61]. Standards for detection of spine fractures have been developed by the Royal Osteoporosis Society, UK [63], but issues around ascertaining the age of spine fractures or any mechanism of injury remain.

The key intervention for an FLS is to get the eligible patients started on anti-osteoporosis medication as soon as possible after the sentinel fracture and reduce the risk of fractures in the imminent risk period [30]. This is especially important for oral AOMs, which may take up to 24 months to achieve maximal efficacy for non-spine fracture prevention [64, 65]. Some national measures are met by initiating investigation and/or treatment within 6 months, such as the US National Committee for Quality Assurance. The initiation of appropriate treatment within 12-16 weeks is more appropriate and should be the goal for which FLSs should aim for given the high risk of imminent fracture in those who have had a sentinel fracture event [30].

It is encouraging that adherence rates for patients seen within the FLS setting are generally higher than those in other populations [66-68]. Another study has shown little difference in adherence at 24 months if an FLS did or did not actively follow up and suggested that longer term adherence should not be a focus for FLSs [69]. However in the realworld setting, recorded persistence of anti-osteoporosis therapy for FLS is sub-optimal and so remains an important component for an FLS [20, 70, 71]. Further, one of the few randomised trials of FLS, which focused on patients with upper limb fractures in the ambulatory setting, demonstrated that the FLS arm had a lower adherence to anti-osteoporosis medication at 24 months compared with usual care [72]. Addressing adherence in the FLS setting is likely to require more than the FLS team providing personalised information about fracture risk and next steps, which failed to improve treatment initiation compared with usual care [73]. For these reasons, the KPIs are focused on the treatment recommendation and persistence with AOM at 16 and 52 weeks, however collected. Future iterations of these KPI may consider treatment recommendation and prescription as separate KPIs. While FLSs have traditionally been primarily focused on AOM, effective prevention of fractures requires that the patient be assessed for falls prevention and recommended evidence-based interventions that reduce falls risk [43, 74]. An indicator for adherence to exercise could be considered for future iterations of the KPI set.

A number of potential indicators were discussed by the group but not included in the proposed KPI set. Fracture or re-fracture rates were not included for a number of reasons. Anti-osteoporosis medications have consistently demonstrated significant fracture reduction both in clinical trials [55] and in the real-world setting [75]. Hence, it is not in question if patients adhering will significantly reduce their fracture risk. The FLS level question is how to best deliver a service that systematically enables high-risk patients to be identified, investigated and treated and adhere to AOMs long enough to achieve this fracture reduction. Further, re-fracture rates are dependent on a number of other patient factors such as age, gender, body mass index, previous fracture history, comorbidities, falls risk and medications that make estimating the expected re-fracture rate at an individual or group level challenging. Patients who are seen again by the FLS having fractured on treatment will often require a different treatment strategy [35].

Another potential KPI was testing for specific laboratory tests. This is pertinent that given that up to $35 \%$ patients with osteoporosis in the FLS setting have secondary causes identified by laboratory testing [76]. While a number of laboratory tests are recommended in a number of national guidelines for secondary fracture prevention, there is little consensus 
between them. An example is vitamin D testing. Given the efficiency of routine supplementation [77] and the complexity of interpreting results in terms of seasonal variation $[78,79]$ and changes with acute inflammation [80], some guidelines pragmatically recommend high-dose empirical supplementation over systematic testing for most patients after a fragility fracture if oral AOMs are recommended [81]. Other studies have questioned the efficacy and safety of higher doses of vitamin D [82], however, highlighting the need to check vitamin D status in many patients. While calcium and vitamin D repletion is indicated for patients requiring AOM in nearly all clinical guidelines, calcium repletion can be readily achieved through diet or using over the counter or prescribed supplements, making measurement of calcium repletion challenging. In addition, clinical studies have demonstrated that in postfracture setting, calcium and vitamin D supplementation alone is insufficient to reduce re-fracture risk [83].

In this initial set of KPIs, Patient Reported Outcome Measures (PROMs) have been omitted due to the potential complexities of provision of tools to support ease of administration. These include potential licensing costs, lack of technical support for patients to complete the required questionnaires, automated uploading of data and systems to analyse PROMs for point of care and real-time analyses. This decision at this time in no way lessons the value of PROMs in personcentred FLSs and work is underway in some countries such as the USA. Indicators that include patient-reported outcomes and experiences will be considered over time as these obstacles are addressed.

We have included preliminary targets for each KPI to allow FLSs to benchmark their service and prioritise components of their service pathway for improvement. Ideally the performance of an FLS should be compared with results from peer FLSs in the same region or country to identify local FLS services that could serve as exemplars for other FLSs and offer mentoring support. For the wider academic community, these KPIs could be used to define an effective FLS and facilitate comparisons between different FLS models of care internationally. If these KPIs are not met, it is unlikely that observed associations between an FLS and re-fracture rates or other outcomes are causal. Future studies to demonstrate the benefits of FLSs on re-fracture rates should only be contemplated after the FLS intervention has demonstrated sustainable achievement in these indicators. We recognise that in some countries, additional indicators may be appropriate to add to reflect regional or national healthcare service models. Such indicators could include the proportion of patients with successful transition to the primary care or community setting for longer term monitoring of AOM.

A common challenge for uptake of these KPIs for FLSs will be the additional burden for collecting, analysing and acting on the KPI within a quality improvement framework. The time and resources to do this will likely lead to the FLS seeing fewer patients initially and have to be balanced with the potential increased effectiveness in service delivery in the medium and long term. Using a similar, but not identical, KPI set in the UK has shown significant gaps in service delivery that could be used to improve patient outcomes locally [20]. For example, efforts to increase identification of patients may be less effective in reducing re-fracture rates locally, compared to ensuring higher levels of treatment recommendations and monitoring in those already identified. The UK experience supports the need for FLSs to integrate service improvement cycles into routine practice and deliver small but transformative steps towards effectiveness and efficiency. National, regional and local stakeholders are recommended to work together to reduce the burden of using these KPIs by providing templates for the minimal clinical dataset, and options for secure direct entry of individual patients and bulk upload of patients as appropriate [19]. The ideal provision would be for the KPI data fields to be integrated within the FLS clinical workflow with (semi)-automatic regular extraction, analysis and reporting. Knowledge of service improvement methodology in healthcare will also be needed for FLS staff [84]. This may require bespoke training as well as forums where FLSs can share both successful and unsuccessful service improvement plans. Models for delivering this are emerging in localities within the FLS community and would benefit from more global coordination [85]. Ideally, absolute standards would be used for all the indicators. However, for some indicators, e.g. KPI 4: DXA within 12 weeks, there is provision for regional or national variation to influence the denominator or numerator, so potentially limit international comparisons, at the expense of the indicator maintaining relevance across different healthcare settings.

\section{Conclusion}

The developed patient-level KPI set will support FLSs to examine their current performance and add service improvement cycles to their routine service provision. It will also permit further benchmarking against FLSs regionally, nationally and internationally. These indicators can also be used by funders and providers when designing, commissioning and monitoring FLSs. The next priorities are to provide the resources for the required data flows and to upskill FLS practitioners to effectively use these indicators in a collaborative quality improvement framework.

Acknowledgements We would like to acknowledge the Falls and Fragility Fracture Audit team at that Royal College of Physicians especially the representation from the Royal Osteoporosis Society, Finbar Martin and David Marsh who championed the FLSDB in the early days as well as past and present members of the FLSDB advisory group. 
Funding information MKJ was supported by the National Institute for Health Research (NIHR) Oxford Biomedical Research Centre (BRC).

\section{Compliance with ethical standards}

\section{Conflicts of interest None.}

Disclaimer The views expressed are those of the authors and not necessarily those of the NHS, the NIHR or the Department of Health.

Open Access This article is licensed under a Creative Commons Attribution-NonCommercial 4.0 International License, which permits any non-commercial use, sharing, adaptation, distribution and reproduction in any medium or format, as long as you give appropriate credit to the original author(s) and the source, provide a link to the Creative Commons licence, and indicate if changes were made. The images or other third party material in this article are included in the article's Creative Commons licence, unless indicated otherwise in a credit line to the material. If material is not included in the article's Creative Commons licence and your intended use is not permitted by statutory regulation or exceeds the permitted use, you will need to obtain permission directly from the copyright holder. To view a copy of this licence, visit http:// creativecommons.org/licenses/by-nc/4.0\%.

\section{References}

1. Briggs AM et al (2019) Integrated prevention and management of non-communicable diseases, including musculoskeletal health: a systematic policy analysis among OECD countries. BMJ Glob Health 4(5):e001806

2. (2018) Global, regional, and national incidence, prevalence, and years lived with disability for 354 diseases and injuries for 195 countries and territories, 1990-2017: a systematic analysis for the Global Burden of Disease Study 2017. Lancet 392(10159):1789_ 1858

3. Kanis JA et al (2008) FRAX and the assessment of fracture probability in men and women from the UK. Osteoporos Int 19(4):385397

4. Marsh D et al (2011) Coordinator-based systems for secondary prevention in fragility fracture patients. Osteoporos Int 22(7): 2051-2065

5. Eisman JA et al (2012) Making the first fracture the last fracture: ASBMR task force report on secondary fracture prevention. J Bone Miner Res

6. Kanis JA, Cooper C, Rizzoli R, Reginster JY, Scientific Advisory Board of the European Society for Clinical and Economic Aspects of Osteoporosis (ESCEO) and the Committees of Scientific Advisors and National Societies of the International Osteoporosis Foundation (IOF) (2019) European guidance for the diagnosis and management of osteoporosis in postmenopausal women. Osteoporos Int 30(1):3-44

7. Blain $\mathrm{H}$ et al (2016) A comprehensive fracture prevention strategy in older adults: the European Union Geriatric Medicine Society (EUGMS) statement. Aging Clin Exp Res 28(4):797-803

8. Lems WF, Dreinhöfer KE, Bischoff-Ferrari H, Blauth M, Czerwinski E, da Silva J, Herrera A, Hoffmeyer P, Kvien T, Maalouf G, Marsh D, Puget J, Puhl W, Poor G, Rasch L, Roux C, Schüler S, Seriolo B, Tarantino U, van Geel T, Woolf A, Wyers C, Geusens P (2017) EULAR/EFORT recommendations for management of patients older than 50 years with a fragility fracture and prevention of subsequent fractures. Ann Rheum Dis 76(5):802-810
9. Khosla S, Hofbauer LC (2017) Osteoporosis treatment: recent developments and ongoing challenges. Lancet Diabetes Endocrinol 5(11):898-907

10. Kim SC, Kim DH, Mogun H, Eddings W, Polinski JM, Franklin JM, Solomon DH (2016) Impact of the U.S. Food and Drug Administration's safety-related announcements on the use of bisphosphonates after hip fracture. J Bone Miner Res 31(8):15361540

11. Michael Lewiecki E et al (2018) Hip fracture trends in the United States, 2002 to 2015. Osteoporos Int 29(3):717-722

12. McLellan AR et al (2003) The fracture liaison service: success of a program for the evaluation and management of patients with osteoporotic fracture. Osteoporos Int 14(12):1028-1034

13. Ganda K, Puech M, Chen JS, Speerin R, Bleasel J, Center JR, Eisman JA, March L, Seibel MJ (2013) Models of care for the secondary prevention of osteoporotic fractures: a systematic review and meta-analysis. Osteoporos Int 24(2):393-406

14. Wu CH, Chen $\mathrm{CH}$, Chen PH, Yang JJ, Chang PC, Huang TC, Bagga S, Sharma Y, Lin RM, Chan DC (2018) Identifying characteristics of an effective fracture liaison service: systematic literature review. Osteoporos Int 29(5):1023-1047

15. Wu CH, Kao IJ, Hung WC, Lin SC, Liu HC, Hsieh MH, Bagga S, Achra M, Cheng TT, Yang RS (2018) Economic impact and costeffectiveness of fracture liaison services: a systematic review of the literature. Osteoporos Int 29(6): 1227-1242

16. Berwick DM (1996) A primer on leading the improvement of systems. Bmj 312(7031):619-622

17. Akesson K, Marsh D, Mitchell PJ, McLellan A, Stenmark J, Pierroz DD, Kyer C, Cooper C, IOF Fracture Working Group (2013) Capture the Fracture: a Best Practice Framework and global campaign to break the fragility fracture cycle. Osteoporos Int 24(8): 2135-2152

18. Javaid MK et al (2015) Effective secondary fracture prevention: implementation of a global benchmarking of clinical quality using the IOF Capture the Fracture(R) Best Practice Framework tool. Osteoporos Int 26(11):2573-2578

19. Javaid MK et al (2017) Fracture Liaison Service Database (FLSDB) annual report: leading FLS improvement: secondary fracture prevention in the NHS, F.a.F.F.A. Programme, Editor. Healthcare Quality Improvement Partnership, London

20. Javaid MK et al (2018) Fracture Liaison Service Database annual report December 2018: achieving effective service delivery by Fracture Liaison Services. Royal College of Physicians, London

21. Marsh D et al (2015) Secondary fracture prevention: first steps to a national audit, R.C.o. Physicians, Editor

22. Ballane G, Cauley JA, Luckey MM, el-Hajj Fuleihan G (2017) Worldwide prevalence and incidence of osteoporotic vertebral fractures. Osteoporos Int 28(5):1531-1542

23. Kanis JA, Johansson H, Odén A, Harvey NC, Gudnason V, Sanders KM, Sigurdsson G, Siggeirsdottir K, Fitzpatrick LA, Borgström F, McCloskey E (2018) Characteristics of recurrent fractures. Osteoporos Int 29(8):1747-1757

24. Barton DW, Behrend CJ, Carmouche JJ (2018) Rates of osteoporosis screening and treatment following vertebral fracture. Spine J

25. van der Velde RY et al (2017) Effect of implementation of guidelines on assessment and diagnosis of vertebral fractures in patients older than 50 years with a recent non-vertebral fracture. Osteoporos Int 28(10):3017-3022

26. Gallacher SJ et al (2007) The prevalence of vertebral fracture amongst patients presenting with non-vertebral fractures. Osteoporos Int 18(2):185-192

27. Pandya J, Ganda K, Ridley L, Seibel MJ (2019) Identification of patients with osteoporotic vertebral fractures via simple text search of routine radiology reports. Calcif Tissue Int 105(2):156-160

28. Lentle B et al (2019) The radiology of osteoporotic vertebral fractures revisited. J Bone Miner Res 34(3):409-418 
29. Kanis J et al (2018) Broken Bones, Broken Lives: a roadmap to solve the fragility fracture crisis in Europe, in Broken Bones, Broken Lives. International Osteoporosis Foundation: http://share. iofbonehealth.org/EU-6-Material/Reports/IOF\%20Report_EU.pdf

30. Pinedo-Villanueva $\mathrm{R}$ et al (2019) Imminent fracture risk assessments in the UK FLS setting: implications and challenges. Archive Osteoporos

31. van den Berg P, van Haard P, Geusens PP, van den Bergh J, Schweitzer DH (2019) Challenges and opportunities to improve fracture liaison service attendance: fracture registration and patient characteristics and motivations. Osteoporos Int 30(8):1597-1606

32. Spechbach H, Fabreguet I, Saule E, Hars M, Stirnemann J, Ferrari S, Rizzoli R, Chevalley T (2019) Higher rates of osteoporosis treatment initiation and persistence in patients with newly diagnosed vertebral fracture when introduced in inpatients than later in outpatients. Osteoporos Int 30(7):1353-1362

33. Densitometry, T.i.S.F.C. (2019) 2019 ISCD Official Positions Adult

34. Cummings SR et al (1993) Bone density at various sites for prediction of hip fractures. The Study of Osteoporotic Fractures Research Group. Lancet 341(8837):72-75

35. Diez-Perez A, Adachi JD, Agnusdei D, Bilezikian JP, Compston JE, Cummings SR, Eastell R, Eriksen EF, Gonzalez-Macias J, Liberman UA, Wahl DA, Seeman E, Kanis JA, Cooper C, IOF CSA Inadequate Responders Working Group (2012) Treatment failure in osteoporosis. Osteoporos Int 23(12):2769-2774

36. NICE (2008) Alendronate, etidronate, risedronate, raloxifene, strontium ranelate and teriparatide for the secondary prevention of osteoporotic fragility fractures in postmenopausal women, in NICE Technology Appraisal guidance 161, N.I.f.H.a.C. Excellence, Editor. NICE, London

37. Conley RB et al (2019) Secondary fracture prevention: consensus clinical recommendations from a multistakeholder coalition. J Bone Miner Res. https://doi.org/10.1002/jbmr.3877

38. Kim J, Hwang JY, Oh JK, Park MS, Kim SW, Chang H, Kim TH (2017) The association between whole body sagittal balance and risk of falls among elderly patients seeking treatment for back pain. Bone Joint Res 6(5):337-344

39. Palumbo $\mathrm{P}$ et al (2016) Predictive performance of a fall risk assessment tool for community-dwelling older people (FRAT-up) in 4 European cohorts. J Am Med Dir Assoc 17(12):1106-1113

40. Podsiadlo D, Richardson S (1991) The timed "up \& go": a test of basic functional mobility for frail elderly persons. J Am Geriatr Soc 39(2):142-148

41. Ensrud KE et al (2019) Association of disease definition, comorbidity burden, and prognosis with hip fracture probability among late-life women. JAMA Intern Med

42. Ahmed LA, Nguyen ND, Bjørnerem Å, Joakimsen RM, Jørgensen L, Størmer J, Bliuc D, Center JR, Eisman JA, Nguyen TV, Emaus N (2014) External validation of the Garvan nomograms for predicting absolute fracture risk: the Tromsø study. PLoS One 9(9):e107695e107695

43. Sherrington $\mathrm{C}$ et al (2019) Exercise for preventing falls in older people living in the community. Cochrane Database Syst Rev 1: $\mathrm{Cd} 012424$

44. NICE (2009) Medicines adherence: involving patients in decisions about prescribing medicines and supporting adherence: Clinical Guideline 76

45. Højsager FD et al (2019) Fracture-induced changes in biomarkers CTX, PINP, OC, and BAP-a systematic review. Osteoporos Int 30(12):2381-2389

46. de Bruin IJA, Wyers CE, van den Bergh J, Geusens PPMM (2017) Fracture liaison services: do they reduce fracture rates? Ther Adv Musculoskelet Dis 9(7):157-164

47. van den Berg P et al (2015) Meeting international standards of secondary fracture prevention: a survey on Fracture Liaison Services in the Netherlands. Osteoporos Int 26(9):2257-2263
48. Newman ED (2011) Perspectives on pre-fracture intervention strategies: the Geisinger Health System Osteoporosis Program. Osteoporos Int 22(Suppl 3):451-455

49. (2019) Effective secondary prevention of fragility fractures: clinical standards for fracture liaison services. Royal Osteoporosis Society

50. Innovation, N.S.W.A.f.C. (2018) Model of care for osteoporotic refracture prevention, M. Network, Editor. ACI, Chatswood

51. Kanis JA, Harvey NC, Cooper C, Johansson H, Odén A, McCloskey E, Advisory Board of the National Osteoporosis Guideline Group (2016) A systematic review of intervention thresholds based on FRAX : a report prepared for the National Osteoporosis Guideline Group and the International Osteoporosis Foundation. Arch Osteoporos 11(1):25

52. Shepstone $\mathrm{L}$ et al (2016) A randomized controlled trial of screening in the community to reduce fractures in older women in the UK (The Scoop Study). Osteoporos Int 27:S1:21

53. Hippisley-Cox J, Coupland C (2012) Derivation and validation of updated QFracture algorithm to predict risk of osteoporotic fracture in primary care in the United Kingdom: prospective open cohort study. BMJ 344(may22 1):e3427

54. Bolland MJ et al (2011) Evaluation of the FRAX and Garvan fracture risk calculators in older women. J Bone Miner Res 26(2):420 427

55. Freemantle N, Cooper C, Diez-Perez A, Gitlin M, Radcliffe H, Shepherd S, Roux C (2013) Results of indirect and mixed treatment comparison of fracture efficacy for osteoporosis treatments: a metaanalysis. Osteoporos Int 24(1):209-217

56. Miller PD, Hattersley G, Riis BJ, Williams GC, Lau E, Russo LA, Alexandersen P, Zerbini CA, Hu MY, Harris AG, Fitzpatrick LA, Cosman F, Christiansen C, ACTIVE Study Investigators (2016) Effect of abaloparatide vs placebo on new vertebral fractures in postmenopausal women with osteoporosis: a randomized clinical trial. Jama 316(7):722-733

57. Javaid MK et al (2016) Fracture Liaison Service (FLS) Database facilities audit. FLS breakpoint: opportunities for improving patient care following a fragility fracture. Royal College of Physicians, London

58. Sanli I, van Helden S, ten Broeke R, Geusens P, van den Bergh J, Brink PRG, Poeze M (2019) The role of the fracture liaison service (FLS) in subsequent fracture prevention in the extreme elderly. Aging Clin Exp Res 31(8):1105-1111

59. von Friesendorff M, Besjakov J, Akesson K (2008) Long-term survival and fracture risk after hip fracture: a 22-year follow-up in women. J Bone Miner Res 23(11):1832-1841

60. (1998) Guidelines for preclinical evaluation and clinical trials in osteoporosis, W.H. Organization, Editor. WHO Library Cataloguing, Geneva

61. Mitchell RM et al (2017) Reporting of vertebral fragility fractures: can radiologists help reduce the number of hip fractures? Arch Osteoporos 12(1):71

62. Fink HA, Milavetz DL, Palermo L, Nevitt MC, Cauley JA, Genant HK, Black DM, Ensrud KE, Fracture Intervention Trial Research Group (2005) What proportion of incident radiographic vertebral deformities is clinically diagnosed and vice versa? J Bone Miner Res 20(7):1216-1222

63. Adams J et al (2017) Clinical guideline for the effective identification of vertebral fractures. Royal Osteoporosis Society

64. Black DM et al (1996) Randomised trial of effect of alendronate on risk of fracture in women with existing vertebral fractures. Fracture Intervention Trial Research Group. Lancet 348(9041):1535-1541

65. Harris ST, Watts NB, Genant HK, McKeever C, Hangartner T, Keller M, Chesnut CH 3rd, Brown J, Eriksen EF, Hoseyni MS, Axelrod DW, Miller PD (1999) Effects of risedronate treatment on vertebral and nonvertebral fractures in women with postmenopausal osteoporosis: a randomized controlled trial. Vertebral 
Efficacy With Risedronate Therapy (VERT) Study Group. JAMA 282(14):1344-1352

66. Senay A, Perreault S, Delisle J, Morin SN, Raynauld JP, Banica A, Troyanov Y, Beaumont P, Jodoin A, Laflamme GY, Leduc S, MacThiong JM, Nguyen H, Ranger P, Rouleau DM, Fernandes JC (2019) Rationale, study design, and descriptive data of the Lucky Bone Fracture Liaison Service. Arch Osteoporos 14(1):19

67. Wu CH et al (2018) Fracture liaison services improve outcomes of patients with osteoporosis-related fractures: a systematic literature review and meta-analysis. Bone

68. Ruggiero C, Zampi E, Rinonapoli G, Baroni M, Serra R, Zengarini E, Baglioni G, Duranti G, Ercolani S, Conti F, Caraffa A, Mecocci P, Brandi ML (2015) Fracture prevention service to bridge the osteoporosis care gap. Clin Interv Aging 10:1035-1042

69. Ganda K, Schaffer A, Pearson S, Seibel MJ (2014) Compliance and persistence to oral bisphosphonate therapy following initiation within a secondary fracture prevention program: a randomised controlled trial of specialist vs. non-specialist management. Osteoporos Int 25(4):1345-1355

70. Dunn P, Webb D, Olenginski TP (2018) Geisinger high-risk osteoporosis clinic (HiROC): 2013-2015 FLS performance analysis. Osteoporos Int 29(2):451-457

71. Chandran M, Cheen M, Ying H, Lau TC, Tan M (2016) Dropping the ball and falling off the care wagon. Factors correlating with nonadherence to secondary fracture prevention programs. J Clin Densitom 19(1):117-124

72. McAlister FA et al (2019) Adherence to osteoporosis therapy after an upper extremity fracture: a pre-specified substudy of the CSTOP randomized controlled trial. Osteoporos Int 30(1):127-134

73. Vaculik J et al (2017) Secondary fracture prevention in hip fracture patients requires cooperation from general practitioners. Arch Osteoporos 12(1):49

74. Gillespie LD et al (2012) Interventions for preventing falls in older people living in the community. Cochrane Database Syst Rev (9): Cd007146

75. Hawley S, Javaid MK, Prieto-Alhambra D, Lippett J, Sheard S, Arden NK, Cooper C, Judge A, REFReSH study group (2016) Clinical effectiveness of orthogeriatric and fracture liaison service models of care for hip fracture patients: population-based longitudinal study. Age Ageing 45(2):236-242
76. Malgo F et al (2016) High prevalence of secondary factors for bone fragility in patients with a recent fracture independently of BMD. Arch Osteoporos 11:12

77. Gallagher JC, Sai A, Templin T 2nd, Smith L (2012) Dose response to vitamin D supplementation in postmenopausal women: a randomized trial. Ann Intern Med 156(6):425-437

78. Hypponen E, Power C (2007) Hypovitaminosis D in British adults at age $45 \mathrm{y}$ : nationwide cohort study of dietary and lifestyle predictors. Am J Clin Nutr 85(3):860-868

79. Bours PHA et al (2011) Seasonal variation of serum 25hydroxyvitamin $\mathrm{D}$ levels in adult patients with inflammatory bowel disease. Osteoporos Int 22(11):2857-2867

80. Reid D, Toole BJ, Knox S, Talwar D, Harten J, O'Reilly DS, Blackwell S, Kinsella J, McMillan D, Wallace AM (2011) The relation between acute changes in the systemic inflammatory response and plasma 25-hydroxyvitamin D concentrations after elective knee arthroplasty. Am J Clin Nutr 93(5):1006-1011

81. Francis R et al (2013) Vitamin D and bone health: a practical clinical guideline for patient management. National Osteoporosis Society

82. Burt LA, Billington EO, Rose MS, Raymond DA, Hanley DA, Boyd SK (2019) Effect of high-dose vitamin D supplementation on volumetric bone density and bone strength: a randomized clinical trial. Jama 322(8):736-745

83. Grant AM, Avenell A, Campbell MK, McDonald A, MacLennan G, McPherson G, Anderson FH, Cooper C, Francis RM, Donaldson C, Gillespie WJ, Robinson CM, Torgerson DJ, Wallace WA, RECORD Trial Group (2005) Oral vitamin D3 and calcium for secondary prevention of low-trauma fractures in elderly people (Randomised Evaluation of Calcium Or vitamin D, RECORD): a randomised placebo-controlled trial. Lancet 365(9471):1621-1628

84. Brandrud AS et al (2017) Domains associated with successful quality improvement in healthcare - a nationwide case study. BMC Health Serv Res 17(1):648

85. Mitchell PJ et al (2019) Quality improvement initiatives in fragility fracture care and prevention. Curr Osteoporos Rep 17(6):510-520

Publisher's note Springer Nature remains neutral with regard to jurisdictional claims in published maps and institutional affiliations. 